\title{
Modern Recognized Methods of Working with Non-Speaking Children of Early Age Internationally
}

\author{
Elena Timofeeva*, Ksenia Timofeeva \\ GAOU VO MGPU, ISO \& KR, 119261 Moscow, Russia
}

\begin{abstract}
This article deals with issues of organization and implementation of correctional care for young children with delayed speech development; we explore the key aspects of brain mechanisms which are fundamental to the child's speech acquisition. Here we describe modern internationally recognized methods (Tomatis, Floortime, Theraplay) which can significantly increase the effectiveness of correctional and pedagogical work with non-speaking children. Substantial attention is paid to issues of parent-child relations.
\end{abstract}

In the modern world, cases of delayed speech development in children make up a significant part among all child development disorders. Search of new effective correction methods is one of the main challenges for both Russian and international academic community in the field of disability studies and speech therapy. This problem relevance is determined by the lack of a common concept which would explain the brain mechanisms instrumental in the course of speech acquisition. While studying speech ontogenesis and dysontogenesis topics, we substantiated the problem with the following theories:

1. L.S. Vygotsky's theory of sensitive periods of development.

2. The brain neuroplasticity theory.

3. Anna Jean Ayres' theory of sensory integration.

4. A. Tomatis' theory on hearing integration.

5. Stanley Greenspan's theory of emotionally significant interaction between the child and the adult.

6. M. Ainsworth and J. Bowlby's theory of attachment.

Tomatis sensorineural auditory stimulation method was created by French ENT doctor and phoniatrist Alfred Tomatis in the 1960s. This method represents a listening-based training system. Alfred Tomatis emphasized that "listening is just as different from hearing as seeing is different from looking" i.e. listening and hearing are completely different physiological processes. Performing specific processing of music and voice, the French scientist has proven that stimulation of the brain through the auditory system not only changes the quality of listening but improves cognitive, emotional and motor abilities of the person. A. Tomatis has explained these improvements by the fact that the ear plays a decisive role in the information processing and is considered a single sensorimotor organ

\footnotetext{
* Corresponding author: timofeevaea402@mail.ru
} 
that provides access to the entire nervous system [1]. The neurophysiological basis of the Tomatis method is the mobilization of middle ear muscles in sessions of listening to music or human voice using the unique TalksUp technology.

Alfred Tomatis' idea regarding the importance of ordering of sensory sensations by the brain (in this aspect, acoustic sensations) chimes with the theory of sensory integration (SI) by A. J. Ayres, who supported the position of sensory feelings (auditive, vestibular, proprioceptive, tactile and visile) serve as "nutrient" for the brain, providing it (the brain) with all necessary knowledge to control the body and thinking [2]. Alfred Tomatis and A. J. Ayres in their studies proved that disordered auditive sensory processes cannot be "digested" by the brain. Accordingly, it is impossible to nourish the brain with them for further training.

We assumed that there is a direct relationship between the delay in speech development at an early age and disorders in the processing of acoustic sensations. Disorganized (nonintegrated) acoustic sensations fail to become the basis for speech development in children of early age. In order to confirm or refute this assumption, an experiment based on the Center for Early Development and Correction "OranzhevoyeDetstvo" ("Orange Childhood", Schelkovo town of Moscow Region) was conducted on 36 children ranging in age from 2 to 4 years. All children had disorders of early speech development while retaining physical hearing. $89 \%$ (32 participants) were boys. There were no children with autism spectrum and genetic disorders among this group. Many children had a mild perinatal pathology in past medical history with subsequent removal of the diagnosis at the age of 1 year. For some of the children, it was not possible to get a their full previous medical records. All of the subjects involved in the experiment were divided into 4 groups:

1. Children with impaired early speech development -2 people $(6 \%)$.

2. Children with motor disabilities and impaired early speech development -8 people $(22 \%)$.

3. Children with cognitive disorders and impaired early speech development - 6 people (17\%).

4. Children with disorders of all developmental lines - 20 people $(55 \%)$.

Only 10 of the 36 children had the basic skills of phrasal speech (2nd level of speech underdevelopment). 26 children were non-speaking. Many of the children had signs of verbal-motor pathology (increased salivation, difficulty in eating, interdental pronunciation of sounds, disturbance of speech breathing). During the experiment, it was also found that most of the children (28 people or $78 \%$ ) had impaired sensory integration, which manifested itself in the form of impaired sensory processing (auditive, vestibular, proprioceptive, tactile and visile). Auditory integration disorders were presented in the following behavioral forms: children didn't always respond to verbal instructions, miscorrelated between the words and subjects/actions. Some children did not understand speech at all. Some of the children could not correctly indicate the source of the sound, were unable to distinguish speech from non-speech noises. Children posessing basics of phrasal speech did not distinguish phonemes similar in sounding, did not understand the prepositional-case constructions. In noisy environments almost all children showed decreased ability for oral-aural perception. Some of the children had an increased sensitivity to non-speech sounds (household items, loud music, a hypermarket).

Based on the theory of Alfred Tomatis about the connection of listening ability with brain training, we assumed that exercises aimed at improving the processing of acoustic sensations can help improve speech skills. Using audio training according to the A. Tomatis method and standard speech therapy exercises, we began to work with impaired auditory integration in our children group of 36 people, dividing them into 2 groups of 18 people. In each group there were children equally developmentally impaired and with an equal level of speech underdevelopment (13 non-speaking children and 5 children with 2 nd level of 
general speech underdevelopment). We organized only speech therapy sessions with a frequency of 2 times a week during a 3 months period in the first group of children. The second group, in addition to the speech therapy, received intensive training classes using the Tomatis method. It consisted of listening to Mozart's music on a daily basis using a multifunctional TalksUp apparatus. One of the functions is the electronic alternation of sound. Through a sharp transition from low frequencies to high frequencies and vice versa, the sound contrast effect is realized, which causes the ear to have an accommodation process. Therefore, the ear stimulates the brain by adapting to changes in sound. In addition, the sound transmission in this therapy is carried out in two ways: 1) through bone conduction; 2) through air conduction. Bone conduction effect was provided by a vibration device built into the headphones. Air conduction was carried out directly through the headphones. Switching of high-low frequencies and vice versa always occurs earlier in bone conduction than in air. Bone conduction is used to prepare the brain to receive, accept and process incoming information that is conducted by air without causing emotional distress.

Thus, all 18 children with speech impairment completed 3 intensive courses of Tomatis therapy in 3 months. 1 course consisted of daily sessions (lasting from 30 to 80 minutes depending on age) for 13 days. Speech therapy sessions continued during the same period.

After 3 months since the beginning of the experiment, we evaluated the children speech abilities in each group and were able to state the following: the group that received only speech therapy (classes 2 times a week) show little improvement in their speech development. In this group, only 2 out of 13 non-speaking children approached the level of "basics of phrasal speech". In the second group, all 13 non-speaking children were already able to construct a short phrase ("Mom, give"; "Come here"). 5 children with the basics of phrasal speech from the first group remained at the same level of speech underdevelopment. From the second group, all children moved to a higher level of speech development (2nd level of GSU). Compared with the first group, the phonetic-phonemic side of speech significantly improved in all children within the second group, and the manifestations of speech-motor pathology decreased. All children from the second group improved their cognitive and motor development, while children from the first group were able to do this to a lesser extent (15\%).

According to the experiment results, it can be stated that all children (18 people) with whom we used the Tomatis hearing training system experienced significant improvements in speech development over a short period of time. Improvements related to both comprehencive and expressive speech, its nominative and predicative functions:

1. improvement of speech understanding (verbal instructions without gesture reinforcement, prepositional sentences);

2. increasement in speech activity (late canonical babble, words, phrasal speech).

In addition, all children of the second group improved non-verbal components of their development (socio-emotional, motor and neurological statuses: behavior, mood, sleep, motor skills and dexterity, self-care and toilet skills).

Thus, we concluded that improving the processing of acoustic sensations significantly accelerates the correction process in children with impaired early speech development. Complementing the classical speech therapy methods with the Tomatis method during the sensitive period of the child's speech development (2-4 years), we not only reduce the total corrective action time, but we can also hope that these children will be able to reach the norm of speech before 7 years.

Our working experience with young children having a speech development delay demonstrates that many non-speaking children have impaired communicative development. This is because one of the main functions of speech is communicative. The search for effective ways and means of correcting speech disorders in this category of children has led 
us to the need of studying the communication and communicative activity topic. We became interested in the topic of parent-child relationships, communication correction and the modern international experience regarding those subjects.

One of scientifically proven methods used in federal and state programs in the United States is so-called Theraplay. The foundation of this therapy was established by Ann M. Jernberg), who led a research on attachment in the 1970's and has proven that interpersonal play is important for children of any age. Today, this Theraplay method is recognized throughout the world and is aimed at healthy socio-emotional (communicative) development of the child. The main principles of the Theraplay method:

- focus on parent-child relationships;

- early intervention, reflecting the importance of early interaction with the parents regarding the child development;

- direct interaction between the adult and the child at the same location and time;

- satisfaction of the child's earlier needs (adaptation to the emotional development level, and not to age);

- interactive communication actively directed by the adults;

- focus on the preverbal, "right-brain" level of development;

- empathy and reflexivity;

- game value, involvement of multiple senses, use of touch.

The goal of therapy is to establish trusting emotional relationship between the child and the parent. The therapeutic process involves 3 stages:

1. Diagnostics:

- standardized questionnaires that address issues of child behavior and parental attitudes;

- initial interview with the parents;

- diagnostics of parent-child relationships using the Marshak Interaction Assessment Method (MIM)

- receiving feedback from parents (a problem is indicated and a therapy contract is concluded).

2. The therapy consists of 6 phases:

- acquaintance

- study

- careful acceptance

- resistance

- growth and trust

- completion

Depending on the problem, parents from the first session can be in the room and participate in the game process together with the therapist, or also can observe what is happening from the observation room. In the second case, another (interpretative) therapist works with parents. If only one therapist is involved in the process, he discusses the sessions with the parents later. The main task of the therapist is to explain to the parent what is happening in the playroom and prepare the parents for active participation in the game with their child.

3. Follow-up observation (control sessions).

In Theraplay-therapies, the key role belongs to the parents. The task of the therapist is to help parents establish a safe, trusting relationship that promotes healthy social and emotional development of the child [3].

Another internationally recognized system aimed to help children with emotional (communicative) disorders is a comprehensive program based on the DIR Method (Developmental, Individual Difference, Relationship-Based). This concept combines 
emotional, social, intellectual and educational challenges faced by the child. Floortime method, an approach developed by Stanley I. Greenspan, exists within this concept. The aim of this method is to form an emotionally significant interaction between the child and the parent in playful/educational situations. Emotional interaction should contribute to the development of the child's nine basic developmental stages. The method's authors call these stages the Functional Emotional Developmental Capacities (FEDCs). Let us consider the first 5 stages corresponding the parameters of interest for us (early age and level of emotional maturity):

Capacity 1: self-regulation and interest in the world. This capacity occurs at the age of 0-3 months and is based on joint attention: the child shows interest and reacts to objects that he sees, hears, touches and other sensory phenomena.

Capacity 2: engaging and relating. This capacity occurs at the age of 2-5 months and is characterized by manifestations of interest in another person, the formation of affection. At this stage of emotional development, the child begins to distinguish between pleasure from interaction with other people and interest in inanimate objects.

Capacity 3: purposeful emotional interaction and two-way communication. It occurs at the age of 4-10 months and is based on various forms of interaction that are accompanied by the expression of emotions, sounds, gestures, and serve to convey the child's intentions.

Capacity 4: shared solving of social problems, mood regulation and development of early self-awareness (10-18 months). This period is particularly important for predictive conclusions about unfavorable ways of emotional development.

Capacity 5: forming symbols, using words and ideas. At this stage (18-30 months), the child is ready for meaningful use of speech, games with adults [4].

In the process of our study, we found that the emotional development of many nonspeaking children at an age of 1.5 - 4 years does not correspond to the $2 \mathrm{nd}$, 3rd, 4th stages of emotional development. Based on this observation, we became interested in analyzing the basic principles of the DIR/Floortime method:

1. A child masters speaking, cogitation and social-emotional skills in the process of relationships, including emotionally significant interaction.

2. Children have different initial abilities to process sensory and motor information.

3. The progress across development areas is interconnected.

Applying these principles, a DIR concept-based correction program is supported by the level of the child emotional development and creation of such game interactions that correspond to his individual features in the sphere of information processing. This program includes not only the Floortime method, but also parental advice, various types of therapy, music lessons, etc. However, Floortime is the central mechanism of the entire development process in the DIR concept. Floortime addresses two key challenges:

1. Follow the child using his natural interests. First, the parent helps the child to do what he wants, then expands the game action scope. As a part of this task, the parent helps the child to interact with objects and people at the same time.

2. Introduce the child into our common world.

The Floortime technique main components are:

- the game session should correspond to the current level of the child emotional development;

- sessions should be held 7 or more times a day;

- duration of a session is from 20 minutes in a day;

- sessions locations may be different (carpet, bath, car, playground);

- sessions are held by a therapist who teaches parents how to engage in play interaction with the child; home sessions are held by parents and siblings, or other children of the same age can be involved. 
Through this process, by studying international methods of correcting communicative development, we find that the problem of emotional disturbances at an early age lies in the field of parent-child relationships. Improving these relationships leads to successful social and emotional development of the child, as well as the formation of the communicative speech function. Inclusion of Theraplay therapy and Floortime method inclusion in speech and language therapy with non-speaking young children is able to significantly increase the effectiveness of correctional and pedagogical work.

\section{References}

1. Alfred A. Tomatis, The Ear and Language (Moulin Publishing Limited, 1996)

2. A. Jean Ayres, Sensory Integration and the Child (Terevinf, Moscow, 2017)

3. Phyllis B. Booth, Ann M. Jernberg, Theraplay: Helping Parents and Children Build Better Relationships Through Attachment-Based Play (Institute of Play-Based Behavioral Therapy, Moscow, 2017)

4. S. Greenspan, S. Wieder, Engaging Autism: The Floortime Approach to Helping Children Relate, Communicate and Think (Terevinf, Moscow, 2018) 